Jaromír KADLEC

Université Palacký d'Olomouc

\title{
PARTICULARITES LEXICALES DU FRANÇAIS DE BELGIQUE
}

\section{Introduction}

À cause des contacts intensifs entre la Belgique francophone et la France, le français de Belgique s'approche $d u$ français de référence mais certaines différences subsistent. Le nombre le plus important de particularités linguistiques $\mathrm{du}$ français de Belgique se situe évidemment au niveau lexical. Pour désigner les spécificités de la langue française en Belgique on emploie généralement le terme belgicisme, mais la définition de cette notion et les critères utilisés pour la constitution des listes de belgicismes ne sont pas homogènes. Dans les belgicismes, il est possible de classer non seulement toutes les différences entre le français de Belgique et le français de référence attestées exclusivement sur le territoire belge mais aussi des phénomènes existant dans le français de Belgique et dans le français parlé dans les régions françaises proches de la Belgique ${ }^{1}$ ou des particularités du français de Belgique attestées également en France où elles sont considérées comme archaïsmes ou ont un emploi limité. De plus, jusqu'à la fin de $\mathrm{XIX}^{\mathrm{e}}$ siècle, la majorité des linguistes étudiant les régionalismes confondaient des spécificités du français de Belgique avec des éléments employés dans le français populaire ou dans les dialectes du français parlés en France.

\section{Domaines d'emploi des belgicismes lexicaux}

On peut rencontrer des belgicismes dans l'administration, au travail, dans l'enseignement, donc dans les domaines où la réalité extralinguistique belge diffère de la France, mais aussi dans la vie quotidienne. Nombreux sont les belgicismes couramment employés dans les médias belges.

La Belgique est composée de trois communautés (flamande, française et germanophone) et dans le français de Belgique, il existe plusieurs mots dérivés du substantif communauté. Outre l'adjectif communautaire qu'on emploie fréquemment aussi en France (surtout en relation avec les Communautés européennes), les verbes communautariser « transférer de la compétence nationale à la compétence des communautés », décommunautariser "transposer de la compétence d'une des communautés à la compétence nationale », le substantif communautarisation « transposition de la compétence nationale à la compétence des communautés » et l'adjectif bicommunautaire " qui se rapporte aux communautés flamande et française » ont été créés en Belgique. À la mairie (maison communale), travaille le maire (bourgmestre ou mayeur, éventuellement maïeur $^{2}$ ) et ses adjoints (échevins ${ }^{3}$ ) chargés d'une partie de l'administration de la

\footnotetext{
${ }^{1}$ La frontière linguistique ne correspond pas à la frontière politique entre les deux pays.

${ }^{2}$ Le substantif mayorat (maïorat) « fonction de maire », " durée de fonction de maire » et l'adjectif mayoral (maïoral) « relatif au maire » ont été dérivés de ce mot.
} 
commune. Le Registre de l'État civil ne remet pas aux nouveaux mariés le livret de famille comme en France mais le livret de mariage. Les communes à régime linguistique spécial sont appelées communes à facilités et la région bilingue de Bruxelles-Capitale est administrée par le ministre-président. Pour désigner une personne qui fait la navette par un moyen de transport collectif entre son domicile et son lieu de travail les Belges emploient le mot navetteur. Les communes ont le droit d'accorder des subventions (subside) et veillent à empêcher le collage des affiches sur d'autres (surcollage). Pour dénoter le périphérique le français de Belgique se sert d'un germanisme ring et une allée carrossable bordée d'arbres est appelée drève.

Certains belgicismes sont utilisés également dans la terminologie économique et juridique, par exemple les substantifs collocation «emprisonnement », « internement de malades mentaux », jobiste " étudiant qui fait un travail rémunéré, généralement occasionnel », les verbes colloquer « emprisonner », « interner un malade mental », jober " exercer un travail occasionnel », péréquater ${ }^{4}$ " adapter au coût de la vie », prester « accomplir un travail » ou les expressions prêter de l'argent « emprunter de l'argent », donner son renon (renom) « résilier le bail ».

Un nombre important de belgicismes est employé dans l'enseignement. Pour désigner l'école maternelle les Belges utilisent les expressions école gardienne ou école frobel et l'institutrice dans l'école maternelle est appelée institutrice gardienne (frobelienne). Les régents ${ }^{5}$ enseignent dans les classes du degré inférieur du secondaire. Le substantif lycée est remplacé en Belgique par le nom athénée. Les études secondaires avec ou sans latin et grec (humanités) ont été remplacées par les études secondaires favorisant les options (rénové). Le substantif student désigne l'étudiant et le nom auditoire la salle de cours. Le kot est une chambre ou un petit appartement loué à un étudiant et les étudiants qui habitent en kot sont appelés koteurs ou cokoteurs (s'il partagent le même kot). Pour désigner l'année universitaire les Belges emploient l'expression année académique. Les études supérieures ne sont pas divisées de la même façon qu'en France et le premier grade universitaire s'appelle candidature. À Louvain-la-Neuve, le substantif prorecteur désigne l'adjoint au recteur tandis qu'à Liège il s'agit du recteur d'université sortant de charge. Plusieurs belgicismes sont présents dans l'argot des étudiants belges. Les étudiants qui redoublent une année sont appelés bisseurs, les étudiants qui sèchent les cours sont brosseurs et pour dénoter le « fayot» on emploie le composé manche-à-balles. La fourche est un temps libre dans un horaire entre deux périodes de cours. ${ }^{6}$ Le substantif copion désigne un document préparé pour la fraude à un examen, la buse ${ }^{7}$ est un échec à un examen, le verbe bloquer ${ }^{8}$ est employé dans le sens de « bûcher » et mofler $^{9}$ dans le sens de « faire échouer à un examen ».

\footnotetext{
${ }^{3}$ On emploie aussi le substantif échevinat « fonction du Conseiller municipal », " services qui dépendent du Conseiller municipal » et l'adjectif échevinal « relatif au Conseiller municipal ».

${ }^{4}$ Dans le français de référence, on n'utilise que le substantif péréquation « rajustement des revenus au coût de la vie ».

${ }^{5}$ Les études qui forment les régents sont appelées régence ou régentat (régendat).

${ }^{6}$ Le verbe fourcher est aussi attesté.

${ }^{7}$ Il existe également le verbe buser « faire échouer à un examen ».

${ }^{8}$ Le nom bloqueur est aussi employé en Belgique.
} 
Dans le français de Belgique, on découvre plusieurs particularités lexicales pratiquement dans tous les domaines de la vie quotidienne. Comme exemple, il est possible de citer des expressions relatives à l'habitation et à l'alimentation. Dans la terminologie d'habitation, on peut rencontrer les belgicismes suivants : maison bel-étage " maison avec rez de chaussée surélevé ", unifamiliale " maison qui n'est destinée qu'à une seule famille », belle place (place de devant) «pièce la mieux meublée où l'on reçoit », posture « statuette », feu ouvert « chemin où l'on brûle des bûches », boiler " chauffe-eau », lessiveuse "10 " machine à laver », calandre " machine à repasser le linge », clenche " poignée de porte ", femme d'ouvrage (femme à journée) « femme de ménage ».

Comme dans les autres variétés de la langue française, il existe un nombre important de spécificités lexicales dans le domaine de l'alimentation. Plusieurs belgicismes désignent des produits de boulanger, par exemple pain intégral « pain complet », pain français « baguette », pain platine « pain cuit dans un moule », cramique " pain au sucre et aux raisins de Corinthe », craquelin « pain au lait et au sucre ", spéculaus « biscuit à la cassonade », etc. La boucherie où l'on vend spécialement de la viande de mouton s'appelle moutonnerie et plusieurs légumes portent des noms différents en France et en Belgique. Comme exemple, on peut citer salade (laitue dans le français de France), salade de blé (mâche dans le français de France), groseille grise ou grosse groseille (groseille à maquereau dans le français de France). Dans le domaine de la gastronomie, on peut mentionner les expressions waterzooi « bouillon où l'on cuit du poulet ou du poisson, qui sont servis dans le bouillon même », maatje « jeune hareng préparé au sel », filet américain " steak tartare », cannibale « toast au steak tartare, à la viande crue » ou caricole (caracole) « escargot ».

\section{Extension géographique des belgicismes et fréquence de leur emploi}

Assez longtemps, les linguistes belges présupposaient une entité « français de Belgique » et ils ne distinguaient pas les belgicismes utilisés dans toute la Belgique francophone de ceux dont l'emploi est géographiquement ou stylistiquement limité. Les belgicismes dont l'usage coïncide avec les frontières de l'État sont appelés les statalismes ${ }^{11}$. Il s'agit essentiellement du vocabulaire administratif tel que accises ${ }^{12}$ " impôts indirects sur le commerce de 1'alcool», athénée « lycée », milicien « celui qui accomplit son service militaire », minimex ${ }^{13}$ « R.M.I. ». La majorité des expressions de l'argot des étudiants est également attestée dans tout le pays. Par contre, certains mots et emplois ont une extension géographique restreinte. Exclusivement dans la région liégeoise, sont employés les mots bonbon " biscuit », chique " bonbon ", bouquette " crêpe faite avec de la farine de sarrasin pure ou mélangée à de la farine de froment » méhin « ennui de santé », balter " plaisanter " ou l'expression avoir pour faire " être dans l'aisance ». L'expression avoir bien le temps ayant la même signification est

\footnotetext{
${ }^{9}$ Le mofleur est un professeur ayant la réputation d'infliger beaucoup d'échecs.

${ }^{10}$ Ce mot était utilisé avant même en France.

${ }^{11}$ Ce terme a été utilisé pour la première fois par Jacques Pohl (1984).

${ }^{12}$ Les Belges emploient aussi le substantif accisien désignant l'agent du service des accises.

${ }^{13}$ Abréviation de l'expression minimum de moyens d'existence.
} 
attestée seulement dans le Hainaut. Plusieurs belgicismes ne sont en usage que dans la région bruxelloise. Il s'agit surtout des expressions empruntées au flamand, comme brol « désordre », snul « individu sans valeur », stoufer " " faire de l'esbroufe », zievereer « bavard impénitent», zinneke « corniaud», ce n'est pas $d u$ spek pour ton bec « c'est trop bon pour toi ». Certains « belgicismes » sont usités aussi en France (essentiellement dans le Nord) ou dans d'autres zones francophones (surtout en Suisse et au Canada). Par exemple le substantif avantmidi « matinée » est attesté aussi au Canada, le nom auditoire " salle de cours » est utilisé également en Suisse et dans certaines régions françaises sont en usage les expressions aubette « kiosque », femme d'ouvrage « femme de ménage » et les numéraux cardinaux septante et nonante.

Certaines différences entre le français de France et le français de Belgique existent aussi dans la fréquence d'emploi de quelques mots. À la différence des Français, les Belges utilisent souvent dans la fonction adverbiale l'adjectif fort qui remplace très employé dans le français de référence. Les Belges emploient couramment le substantif subside "subvention », attesté dans les dictionnaires français mais rarement employé. Tandis que les Français installent le chauffage ou l'électricité, les Belges les placent. Dans le français de Belgique, on utilise fréquemment le nom entièreté qui est plutôt rare en France parce que les Français préfèrent le substantif totalité. Plusieurs mots n'ont pas les mêmes connotations en France et en Belgique. En général, on peut dire que les Belges trouvent certaines expressions françaises trop familières ou même grossières. Par exemple, les substantifs époux et épouse qui appartiennent en France plutôt au style administratif remplacent en Belgique dans la conversation quotidienne les mots mari et surtout femme que les Belges ne trouvent pas suffisamment polis. Les injures de type imbécile ou con qui entrent en France de plus en plus dans l'usage familier de locuteurs bien instruits, demeurent en Belgique très grossières.

\section{Formation de nouvelles unités lexicales dans le français de Belgique et belgicismes sémantiques}

Le français de Belgique évolue - si on néglige les emprunts qui n'existent que dans cette variété de la langue française - de la même façon que le français de référence. Pour former de nouvelles unités lexicales les Belges se servent de mêmes affixes que les Français. À l'aide de ces préfixes et suffixes, le français de Belgique a créé des mots non attestés dans le français de référence. Dans le domaine de la suffixation, il est possible de citer les substantifs aidant « celui qui aide », ardoisier « couvreur », autocariste " conducteur d'autocar », " patron d'une compagnie d'autocars », dîneur « celui qui prend le rapas de midi », échasseur " homme monté sur des échasses », exercicier " cahier d'exercices », houillerie " exploitation des mines de charbon », jeteuse " cartomancienne », laverie « buanderie », légistique « action d'élaborer des lois », légumier ou verdurier « marchand de légumes », machiniste « mécanicien », « conducteur de locomotive », myrtillier « arbuste produisant des myrtilles », pigeonniste « colombophile », plafonneur « plâtrier ", seniorie " résidence pour personnes

\footnotetext{
${ }^{14}$ Le substantif stoefer désigne l'homme qui fait de l'esbroufe.
} 
âgées », siroperie ${ }^{15}$ « fabrique de sirop », subsidiation « action de subventionner », les adjectifs amitieux «amical », exemplatif « relatif à l'exemple », principautaire $^{16}$ " relatif à la principauté », rattachiste « favorable au rattachement de la Wallonie à la France », subsidiable « qui peut être subventionné », l'adverbe exemplativement « d'une manière exemplative " ou le verbe subsidier «subventionner ». Les préfixes ont permis de former par exemple les substantifs décumul « séparation fiscale des revenus d'un couple », défusion " réinstauration de l'autonomie des communes qui avaient été fusionnées», méconduite « mauvaise conduite », les verbes adire " convaincre », décumuler " séparer du point de vue fiscal », défusionner " réinstaurer l'autonomie des communes qui avaient été fusionnées », se méconduire «se conduire mal » ou prépensionner ${ }^{17}$ « mettre en préretraite ».

Dans la région bruxelloise, le français se sert souvent du préfixe ver-emprunté au flamand. Comme exemple, on peut citer les verbes veroublier « oublier », verexpliquer « expliquer ». Dans le français populaire de Bruxelles, on emploie fréquemment - à cause de l'influence flamande - les diminutifs, par exemple l'expression le petit manneke pis $^{18}$ " garçon » qui est pléonastique parce que le suffixe a déjà le sens diminutif. L'élément -je (-tje) est aussi d'origine flamande. Le substantif ket, qu'on emploie aussi en Wallonie désigne le garçon et le nom ketje le petit garçon bien que les deux mots soient utilisés aujourd'hui souvent de manière synonymique. Le petit verre s'appelle pintje.

Certains verbes sont utilisés en Belgique - à la différence du français de référence - avec un préfixe, par exemple $s$ 'abaisser ${ }^{19}$ (baisser dans le français de référence), ac(c)onduire (conduire dans le français de référence), soulever un lièvre (lever un lièvre dans le français de référence). Par contre le verbe redoubler est employé en Belgique sans préfixe (Il double sa classe). ${ }^{20}$ Quelquefois, le français de Belgique et le français de référence n'emploient pas les mêmes affixes, par exemple rabattre les oreilles (rebattre les oreilles dans le français de référence), affiler (enfiler dans le français de référence), chamoisette (chamoisine dans le français de référence), minorisation (minoration dans le français de référence), rancuneux (rancunier dans le français de référence).

Dans le français de Belgique, les mots composés se forment de la même manière que dans le français de référence. Parmi les composés créés dans la variété belge de la langue française, on peut citer les substantifs accroche-pied « croche-pied », après-quatre-heures « partie de la journée entre le goûter et le repas du soir », avant-plan « premier plan », bel-étage " rez-de-chaussée surélevé », brise-vue « brise-bise », carte-vue « carte postale », court-vite (va-vite) "diarrhée », demi-doux « personne un peu simple d'esprit », frotte-manche " flagorneur », chef-garde " contrôleur aux chemins de fer », chemin-de-ferriste « cheminot », lèche-plat « raclette de cuisine », mêle-tout « personne qui se mêle

\footnotetext{
${ }^{15}$ On emploie aussi le nom siropier « fabricant de sirop ».

${ }^{16} \mathrm{Ce}$ mot désigne, avec une connotation péjorative, les habitants de Liège.

${ }^{17}$ Les substantifs prépension « préretraite » et prépensionné « préretraité » sont également attestés.

${ }^{18}$ Il existe aussi des variétés menneke, manèquet, maniquet.

${ }^{19}$ En plus, ce verbe est pronominal, par exemple Abaisse-toi un peu, que je vois ce qui est devant moi!

${ }^{20}$ Le substantif doubleur désigne en Belgique un élève qui redouble la classe.
} 
de tout », porte-paquet « porte-bagages », tapis-plein « moquette », toute-boîte « imprimé gratuit distribué dans toutes les boîtes aux lettres ».

Certains mots n'ont pas le même sens dans le français de Belgique et dans le français de référence. Il s'agit par exemple des belgicismes sémantiques suivants : banlieu « omnibus », bouger à « toucher à », calepin « cartable d'écolier », cour « toilettes », doubleur " élève qui redouble la classe », farde "chemise », « dossier », griffe « égratignure », lycée ${ }^{2 l}$ " école secondaire pour jeunes filles », mallette « cartable », pantoufle « chaussure de toile pour la gymnastique », pistolet « petit pain rond », place « pièce d'habitation », préfet « directeur d'un lycée », proviseur « adjoint au directeur d'un lycée », quartier « petit appartement », ramassette " pelle à poussière ", torchon « serpillière », vidange " récipient consigné ».

\section{Archaïsmes}

Les Belges emploient plusieurs expressions considérées en France comme archaïques et le conservatisme est l'un des traits caractéristiques du français de Belgique. En général, il est vrai que le nombre d'archaïsmes est directement proportionnel au niveau d'isolation et d'éloignement géographique par rapport à la métropole. Pour cette raison, le français de Belgique est beaucoup moins riche en archaïsmes que les français québécois et acadien. Le substantif clignoteur, utilisé autrefois même en France, désigne en Belgique toujours le dispositif servant à indiquer la direction que va prendre un véhicule tandis que le français de France préfère aujourd'hui le nom clignotant. Parmi les autres archaïsmes présents dans le français de Belgique, on peut citer les substantifs aubette ${ }^{22}$ " kiosque », gazette « journal », lessiveuse « machine à laver », malle « bateau », minerval « droit de fréquentation de certaines écoles », nonnette ${ }^{23}$ " nonne », roulage « circulation routière », l'adjectif cru « climat froid et humide » ou les verbes épastrouiller « étonner fortement », courtiser « être fiancé ». L'emploi des mots déjeuner, dîner et souper pour désigner les repas de matin, de midi et de soir, existant aussi dans des autres variétés de la langue française, est également archaïque.

Le vocabulaire juridique belge est encore plus archaïque que le vocabulaire juridique français. Au lieu du verbe enquêter, les Belges emploient indaguer, au lieu de l'expression il résulte (de la procédure) ou il appert (des documents), on utilise en Belgique il conste et au lieu du substantif pertinence et de l'adjectif pertinent on peut rencontrer relevance (les faits sans relevance dans notre affaire) et relevant (faits relevants). ${ }^{24} \mathrm{Il}$ existe encore d'autres archaïsmes dans le français juridique de Belgique, par exemple le substantif prescrit (conformément au prescrit de l'article 5) « ce qui est prescrit» ou le verbe comminer (Le conseil communal peut comminer des peines contre les infractions) « prononcer ».

\footnotetext{
${ }^{21}$ Ce mot a été utilisé jusqu'à l'instauration de la mixité dans l'enseignement.

${ }^{22}$ L'Académie française recommande ce mot pour remplacer le substantif abribus.

${ }^{23}$ Ce substantif est utilisé dans le « français familier ».

${ }^{24}$ Emploi que l'on trouve chez Bossuet.
} 


\section{Emprunts}

Pour des raisons géographiques et historiques, il existe assez de différences entre le français de Belgique et le français de référence dans le domaine des emprunts. Le français de Belgique a emprunté plus de mots à l'allemand et surtout au flamand que le français de référence. Le règne des Habsbourg espagnols a influencé aussi le lexique du français parlé en Belgique et les Belges emploient certains emprunts à l'espagnol que les Français ne connaissent pas. Plusieurs mots ont été empruntés à l'anglais et il ne faut pas oublier les emprunts aux langues régionales parlées sur le territoire belge (surtout au wallon et au picard), issues comme le français de référence de la langue d'oïl.

Le français de Belgique a emprunté aux langues régionales des mots ainsi que des locutions. Plusieurs emprunts sont utilisés par la majorité de la population francophone de Belgique. Il s'agit par exemple des substantifs archelle " étagère ", cacaille " objet sans valeur», chantoir "bétoire», cumulet « culbute », ducasse « fête patronale », escabelle « échelle double », flatte «bouse de vache », «béret de certains groupes d'étudiants», gosette « chausson aux fruits », place « pièce d'habitation », rawette ${ }^{25}$ «petit supplément gratuit », des verbes ravoir " prendre sa revanche sur qqn », et se ravoir " retrouver sa condition physique normale après une émotion » ou d'une locution prendre le café « manger vers 16 heures ». Parmi les expressions dont l'emploi est géographiquement limité, on peut citer par exemple raccuser « cafarder », grandiveux « hautain », ne pas faire de bien « être inquiet», avoir bien le temps « être dans l'aisance », aller se faire enrager « aller au diable ».

Dans le français de Belgique, il existe aussi des emprunts au latin non attestés dans le français de référence, par exemple culpeux « délictuel », vinculer " réduire à l'impuissance ». Les Belges utilisent également la locution latine qualitate qua, remplacée dans le français de référence par l'expression ès qualités. Dans l'enseignement et dans l'administration, on emploie valves ou ad valvas « tableau d'affiche » et minerval « droit de fréquentation de certaines écoles ».

Les emprunts à l'espagnol apparaissent dans le français parlé sur le territoire de la Belgique d'aujourd'hui aux XVII ${ }^{\mathrm{e}}$ et XVIII ${ }^{\mathrm{e}}$ siècles. L'emploi de la majorité de ces emprunts n'était qu'occasionnel et éphémère, à l'exception de quelques mots comme amigo "prison de police», caracole « escargot», escavèche « poisson en gelée vinaigrée », qui sont employés en Belgique même aujourd'hui. Parmi les emprunts plus récents, on peut citer le substantif bodega « débit de boissons », considéré en Belgique comme vieilli. En général, il faut dire que le nombre d'emprunts à l'espagnol dans le français de Belgique et dans le wallon est très restreint et leur importance est souvent surestimée.

L'influence de l'allemand sur le français de Belgique reste assez limitée. Les Belges ont emprunté à l'allemand par exemple les mots froebel " ${ }^{26}$ "école maternelle », ring " périphérique », maitrank «boisson constituée de vin blanc additionné de cognac dans lequel ont mariné des fleurs d'aspérule odorante ». Il serait très intéressant d'étudier l'influence de l'allemand et des langues germaniques sur le français de Belgique et sur le tchèque. Dans plusieurs cas, on

\footnotetext{
${ }^{25} \mathrm{Ce}$ mot peut désigner aussi un enfant dernier-né, venu longtemps après les autres.

${ }^{26}$ Le mot a été derivé du nom de pédagogue allemand Froebel.
} 
s'aperçoit que le français de Belgique dispose d'emprunts non attestés dans le français de référence, mais utilisés dans les langues slaves. On peut citer, par exemple année académique (akademický rok), liberté académique (akademická svoboda), quart d'heure académique (akademická čtvrthodina), student (student), logopédie (logopedie), assiette profonde (hluboký talír), dringuelle (tringelt), prober (prubnout), etc.

Les rapports entre les francophones et les néerlandophones et le bilinguisme d'une partie de la population belge ont créé des conditions favorables pour les emprunts entre les deux langues. Dans les emprunts au flamand, il faut distinguer les expressions proches du flamand par leur forme, considérées comme bruxelloises et utilisées surtout dans le parler appelé brusselaire, des unités beaucoup mieux intégrées dans le système de la langue française et employées sur tout le territoire belge. Dans le premier groupe, on peut classer par exemple les mots boestring «hareng fumé », brol « désordre », broubeler ${ }^{27}$ " bredouiller», kapstok " porte-manteau », kœkebak « crêpe », krotje " petite amie », platekees « fromage blanc», rammeling « raclée », snul « imbécile», sukkeleer ${ }^{28}$ " pauvre type », zwanze 29 «type d'humour propre à Bruxelles» ou les expressions en stoemelings " en cachette », faire de son jan (stouf, stoump) « faire de l'esbroufe », avoir un dikkenek ${ }^{30}$ " être prétentieux » ou les locutions calquées avoir des ruses avec quelqu'un (faire des ruses à quelqu'un) " avoir des ennuis avec qqn », faire de son nez "faire des embarras», ne pas savoir de chemin avec quelqu'un «ne pas savoir comment s'y prendre avec qqn », tenir le fou avec quelqu'un « se payer la tête de qqn», tirer son plan «se débrouiller». Dans le deuxième groupe, appartiennent les mots bourgmestre ${ }^{31}$ «maire», couque «biscuit», «pain d'épices », crolle $e^{32}$ « copeau », « boucle de cheveux », cron, cronte « courbé », dringuelle «pourboire» «argent de poche», kermesse $e^{33}$ "fête partonale», minque « halle aux poissons » ou les calques cour « toilettes » et école gardienne « école maternelle». Plusieurs flandricismes s'intègrent dans le système grammatical français et créent les formes féminines, par exemple baes (baas) / baesine (bazine), "patron»/ "patronne». Certains emprunts au flamand ont contribué au maintien des archaïsmes français. Sous l'influence du verbe flamand presteren, on emploie par exemple en Belgique toujours le verbe prester « accomplir un travail ».

Le français de Belgique subit - comme le français de référence - l'influence de l'anglais. Le patriotisme belge est moins fort que le patriotisme français et l'anglophobie et la révolte contre la culture américaine n'y sont pas très développées. Pour ces raisons, les attaques contre les anglicismes sont moins fréquentes en Belgique qu'en France. Les différentes attitudes envers les anglicismes sont dues aussi au fait que la Belgique n'est pas - à la différence de la

\footnotetext{
${ }^{27}$ Le substantif broubelaire « celui qui bredouille » a été dérivé de ce verbe.

${ }^{28}$ Il existe aussi le verbe sukkeler « travailler dur sans beaucoup de profit ».

${ }^{29}$ Le verbe zwanzer et le substantif zwanzeur sont également attestés.

${ }^{30}$ Le calque avoir un gros cou a le même sens.

${ }^{31} \mathrm{Ce}$ mot est attesté, à l'exception de la zone où on parle le gaumais, dans toute la Belgique. Dans la zone de gaumais, on dit maire et le mot bourgmestre n'apparait que dans les documents officiels.

${ }^{32}$ Il existe aussi le verbe croller « boucler » qui fait partie de la locution son nez crolle après quelque chose « il a envie de ».

${ }^{33}$ Le mot est employé occasionnellement aussi en France.
} 
France - le pays monolingue. Les deux principales communautés linguistiques belges se réunissent rarement dans la lutte contre l'anglais. Dans la majorité des cas, l'anglais est un allié dans le combat contre le français (dans la Région flamande) ou contre le flamand (en Wallonie). Il existe aussi un concept selon lequel l'anglais pourrait créer un pont entre les francophones et les néerlandophones en Belgique et plusieurs Belges (surtout les jeunes) communiquent entre eux dans cette langue. Cette idée est défendue surtout par les Flamands tandis que les francophones rendent les Flamands responsables de la pénétration des anglicismes dans le français de Bruxelles où on peut voir couramment des affiches type Men's Kwaf, Fun Kwaf, New Tif, Tart'Inn et attirent l'attention sur la position dominante de l'anglais aux Pays-Bas.

Il existe une différence importante entre la France et la Belgique dans la fréquence d'emploi des anglicismes. Pour désigner les joueurs de football les Français emploient les mots gardien, arrière, milieu et la fréquence d'emploi des anglicismes keeper, back, half est aujourd'hui extrêmement restreint ${ }^{34}$ tandis que les Belges emploient ces anglicismes couramment. Les «supporteurs » entendent les anglicismes goal et half-time beaucoup plus souvent en Belgique qu'en France où ils sont considérés archaïques et les expressions kick-off ou throw-in ne s'emploient qu'en Belgique. Même la prononciation des anglicismes diffère souvent. Les Belges prononcent au début du mot iceberg [aj] tandis qu'en France la pronciation [aj] et [i] est attestée. Le mot steward est prononcé en Belgique de la même manière qu'en anglais mais les Français prononcent en général [stiwaRt]. Des différences similaires existent dans la terminologie militaire, essentiellement dans l'aviation et dans la marine. La marine belge emploie le mot midship tandis que les Français privilègient les substantifs aspirant et enseigne. Dans l'aviation belge, on utilise fréquemment le mot wing mais les Français préfèrent l'expression escadre. À la différence de la France, l'emploi des anglicismes est beaucoup moins limité par des mesures administratives et pour cette raison il est possible de rencontrer dans les magasins belges les anglicismes comme after-shave (aprèsrasage dans le français de référence) ou body-milk (lait de beauté dans le français de référence). Les agences de voyages belges offrent le minitrip «petit voyage organisé ». Parmi les autres anglicismes couramment employés en Belgique, on peut mentionner home, "résidence», full time (part time) «temps complet» ( « mi-temps »), caddie « chariot», panty «collant» ou shifter ${ }^{35}$ " relayer une personne ou une équipe à la fin d'une période de travail ». Il existe aussi des anglicismes attestés seulement en Belgique, comme jobiste « étudiant qui fait un travail rémunéré, généralement occasionnel » ou flat «studio», "petit appartement». Les Belges ont formé aussi quelques pseudoanglicismes, qui semblent empruntés à l'anglais mais ne sont pas attestés dans les pays anglophones. C'est le cas de fancy-fair, tramway-man, taxi-man ou des termes désignant les joueurs ou les «supporteurs» des clubs de football en Belgique (Sportingman, Racingman, Standardman, Antwerpman). ${ }^{36}$ Il y a une grande différence dans l'emploi des anglicismes entre la Wallonie et Bruxelles où les

\footnotetext{
${ }^{34}$ Ces mots n'apparaissent même pas dans le dictionnaire Le Nouveau Petit Robert.

${ }^{35}$ Ces expressions sont employées surtout dans la marine et dans l'industrie pétrolière.

${ }^{36}$ Les pseudoanglicismes existent également en France, comme par exemple tennisman ou rugbyman (tennis et rugby player en anglais).
} 
contacts entre le français et l'anglais sont beaucoup plus fréquents et le dernier y joue le rôle d'une langue neutre dans les situation quand il faut choisir entre le français et le néerlandais. Bruxelles voudrait devenir le centre multiculturel et cosmopolite de l'Europe, donc endroit idéal pour la diffusion de l'anglais. En plus, les communautés anglaises et américaines, assez nombreuses, avec leurs écoles et centres culturels sont présentes dans la ville. Mais pour l'instant, il paraît que l'influence de l'anglais dans la capitale belge n'est pas très importante et l'anglais ne menace pas - si on néglige la publicité et l'affichage en anglais présents dans toutes les grandes villes européennes - la position de la langue française. Bruxelles est le siège de plusieurs institutions européennes et peut donner l'impression que l'anglais y est fréquemment employé mais cette impression est souvent fausse.

\section{Conclusion}

Il existe toujours des différences entre le lexique du français de Belgique et le français de référence bien que les deux systèmes approchent progressivement. Le vocabulaire du français de Belgique est influencé en Wallonie par le wallon et les autres langues régionales parlées dans la Belgique francophone et dans la zone bilingue bruxelloise par le néerlandais. Comme dans toutes les variétés non métropolitaines de la langue française, plusieurs archaïsmes et dialectalismes sont attestés dans le français de Belgique. Les Belges ont aussi un rapport aux anglicismes différent de celui des Français.

\section{BIBLIOGRAPHIE}

BAETENS BeARDSMORE Hugo (1979), Le contact des langues à Bruxelles. Dans: VALDMAN Albert, Le français hors de France, Paris, Champion, p. 223-247.

Bal Willy, DopPagne Albert, Goosse André, Hanse Joseph, LenOBLe-Pinson Michèle, POHL Jacques, WARNANT Léon (1997), Belgicismes, inventaire des particularités lexicales du français en Belgique, Louvain-la-Neuve, Duculot.

Blampain Daniel, Goosse André, Klinkenberg Jean-Marie, Wilmet Marc (1999), Le français en Belgique, Bruxelles, Duculot.

Couvreur Michèle, DezutTer Olivier, Vervier Anne (1992), Entendons-nous, Bruxelles, Didier Hatier.

DopPagne Albert (1979), Belgicismes de bon aloi, Bruxelles, Fondation Charles PlisnierOffice du bon langage.

FRANCARD Michel (1993), Entre Romania et Germania : la Belgique francophone. Dans : ROBILLARD Didier de, BENIAMINO Michel, Le français dans l'espace francophone I, Paris, Honoré Champion, p. 317-336.

GoOsSE André (1977), Qu'est-ce qu'un belgicisme ?, Bulletin de l'Académie royale de langue et littérature françaises 55, p. 345-367.

GoOsse André (1984), Influences de l'anglais sur le français de Belgique, Cahiers de l'Institut linguistique de Louvain 9, p. 1-2.

LEBOUC George (1998), Le belge dans tous ses états (dictionnaire de belgicismes, grammaire et prononciation), Paris, Bonneton.

LENOBLE-PINSON Michèle (1991), Anglicismes et substituts français, Louvain-la-Neuve, Duculot. 
MiLIČKOVÁ Ladislava (1996), Le parler français de Belgique I, Sborník prací Filozofické fakulty brněnské univerzity L 17, Brno, Masarykova univerzita, p. 7-15.

MiLIČKOVÁ Ladislava (1997), Le parler français de Belgique II, Sborník praci Filozofické fakulty brněnské univerzity L 18, Brno, Masarykova univerzita p. 53-60.

PETŘíKOVÁ Jarmila (1992), La Belgique et le français belge, AUPO-Romanica 4, Philologica 63, Olomouc, Univerzita Palackého, p. 101-105.

PIRON Maurice (1973), Les belgicismes lexicaux : essai d'un inventaire, Travaux de linguistique et de littérature, XI, 1, Strasbourg, p. 295-304.

PIRON Maurice (1979), Le français de Belgique. In : VALDMAN Albert, Le français hors de France, Paris, Champion, p. 201-221.

POHL Jacques (1984), Le statalisme, Travaux de linguistique et de littérature 22, p. 251264.

PRIESOLOVÁ Janka (2002-2003), Lexikální zvláštnosti belgické francouzštiny, Cizí jazyky 46, p. 156-158.

ŠABRSUlA Jan (1966-1967), Belgická francouzština, Cizi jazyky ve škole 10, p. 193-195.

ŠABRSUlA Jan (1980), Znovu k belgické francouzštině, Cizi jazyky 24, p. 96. 


\begin{abstract}
The article focuses on the lexical particularities of the French language used in Belgium. Even if the French used in France and the French used in Belgium are successively converging, certain differences in the vocabulary are still observable. In Wallonia, Belgian French is influenced especially by Walloon and by other Romance languages and dialects used in this territory and in the bilingual Bruxelles, it is influenced by Dutch. As in other regional varieties of French, the archaic and dialectal units may be found in Belgian French. The Belgians also have a somewhat different approach to anglicisms than the French.
\end{abstract}

